\title{
Symmetry Parameters of CSS Sources: Evidence of Fuelling?
}

\author{
D. J. Saikia ${ }^{1}$, S. Jeyakumar ${ }^{2}$, F. Mantovani ${ }^{3}$, C. J. Salter ${ }^{4}$, \\ R. E. Spencer ${ }^{5}$, P. Thomasson ${ }^{5}$ and P. J. Wiita ${ }^{6}$ \\ ${ }^{1}$ NCRA, TIFR, Post Bag No. 3, Ganeshkhind, Pune 411 007, India \\ djs@ncra.tifr.res.in \\ ${ }^{2}$ Physikalisches Institut, Universität zu Köln, 50937 Köln, Germany \\ jeyaks@ph1.uni-koeln.de \\ ${ }^{3}$ Istituto di Radioastronomia, CNR, Via P. Gobetti 101, I-40129 Bologna, Italy \\ fmantova@ira.cnr.it \\ ${ }^{4}$ Arecibo Observatory, HC3 Box 53995, Arecibo, PR 00612, USA \\ csalter@naic.edu \\ ${ }^{5}$ Jodrell Bank Observatory, Macclesfield, Cheshire SK11 9DL, UK \\ pt@jb.man.ac.uk, res@jb.man.ac.uk \\ ${ }^{6}$ Georgia State University, Atlanta GA 30303-3083, USA \\ wiita@chara.gsu.edu
}

Received 2002 July 18, accepted 2002 December 9

\begin{abstract}
The compact steep spectrum (CSS) and gigahertz peaked spectrum (GPS) sources are widely believed to be young radio sources, with ages $\lesssim 10^{6} \mathrm{yr}$. If the activity in the nucleus is fuelled by the supply of gas, one might find evidence of this gas by studying the structural and polarisation characteristics of CSS sources and their evolution through this gas. In this paper we discuss some of the possible 'smoking-gun' evidence of this gas which may have triggered and fuelled the radio source.
\end{abstract}

Keywords: galaxies: active — quasars: general — radio continuum: galaxies

\section{Introduction}

There is a consensus of opinion that the compact steep spectrum (CSS) and gigahertz peaked spectrum (GPS) sources are young objects seen at an early stage of their evolution. Recent measurements of component advance speeds for a few very compact sources yield ages of about $10^{3}$ yr (Owsianik \& Conway 1998; Taylor et al. 2000). Spectral studies of CSS sources suggest ages $\lesssim 10^{5}$ yr (Murgia et al. 1999). Models have been constructed for the evolution of extragalactic radio sources from the most compact symmetric objects, christened CSOs, to the medium symmetric objects (MSOs) and later on to the standard FR II radio sources (e.g. Carvalho 1985; Fanti et al. 1995; Readhead et al. 1996; O’Dea 1998 and references therein). Numerical simulations of jet propagation can reproduce these evolutionary steps (e.g. De Young 1997). Evolutionary scenarios for CSOs constrained by their self-similar evolution (Jeyakumar \& Saikia 2000) and dependence of radio luminosity on linear size (Snellen et al. 2000) have also been explored (Perucho \& Martí, 2002)

An interesting related question is the fuelling of these young radio sources, possibly due to the infall of gas to the central regions due to interacting companions and mergers. Detailed calculations (e.g. Hernquist \& Mihos 1995) showed that following a merger the infall of interstellar matter into the central few hundred pc takes place over a time scale of $\approx 10^{8} \mathrm{yr}$. In such a situation, it should be possible to find evidence of this infalling material by following the evolution of the radio components in these young radio sources as they advance outwards and interact with this material.

\section{Symmetry Parameters}

\subsection{Arm-Length and Flux Density Ratio}

One straightforward test is to examine the symmetry parameters, namely the arm-length or separation ratio, $r_{\mathrm{D}}$, of the outer lobes from the nucleus, and the flux density ratio, $r_{\mathrm{L}}$, of the lobes. In order to estimate reliably the separation ratio, $r_{\mathrm{D}}$, one also needs to identify the radio core or nuclear component. Of the initially symmetric but oppositely-directed radio lobes, the one propagating in the direction of more infalling material will encounter a denser ambient medium, and thus should be both brighter and closer to the nucleus. However, this test is unlikely to be a sharp one because the infalling material or a merging galaxy is likely to cover only a relatively small solid angle, depending on its size and separation from the nucleus. The symmetry parameters would also depend on the orientation, bulk relativistic motion and evolution of the individual components.

The distributions of $r_{\mathrm{D}}$, defined to be $>1$, for a sample of 109 FR II sources from the 3CRR and S4 samples of radio sources with a radio luminosity at $178 \mathrm{MHz} \gtrsim 10^{26} \mathrm{~W} \mathrm{~Hz}^{-1} \mathrm{sr}^{-1}\left(H_{\mathrm{o}}=100 \mathrm{~km} \mathrm{~s}^{-1} \mathrm{Mpc}^{-1}\right.$ and $q_{\mathrm{o}}=0$ ) and a detected radio core are shown in Figure 1. A CSS source is defined to be $<20 \mathrm{kpc}$ in size. However, any limiting value is likely to be somewhat ad hoc, and one could sometimes find evidence of interactions in larger sources as well. The CSS sources associated with 

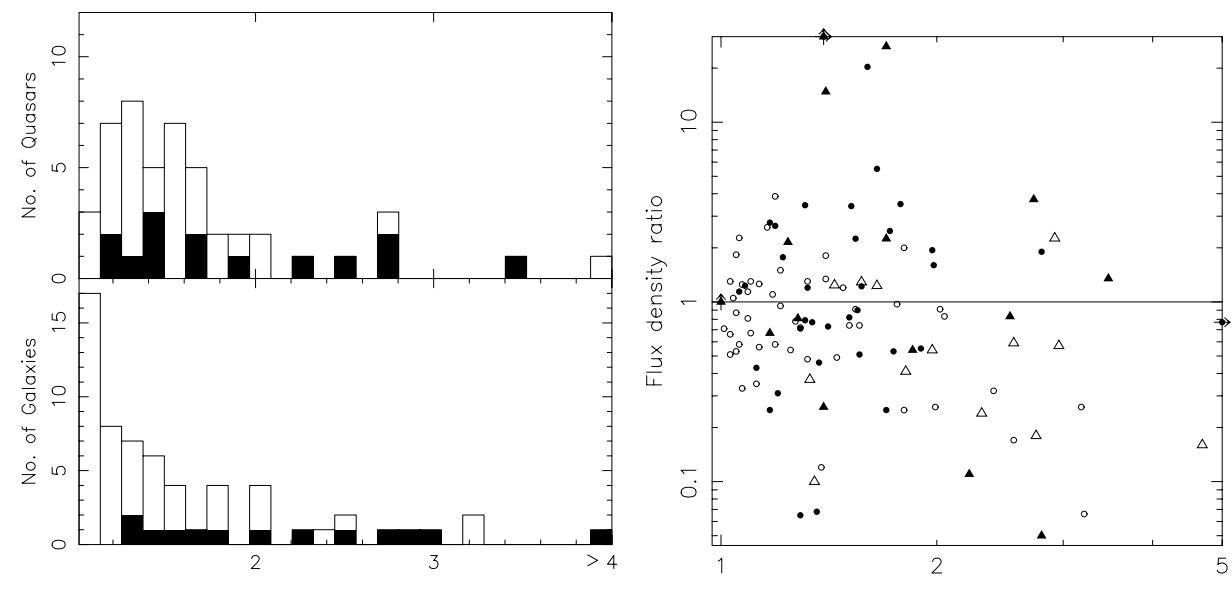

Separation ratio

Figure 1 Left: The distributions of the arm-length or separation ratio, $r_{\mathrm{D}}$, for the sample of high-luminosity FR in radio galaxies and quasars. The CSS sources are shown in black. Right: The $r_{\mathrm{D}}-r_{\mathrm{L}}$ diagram, where the filled and open symbols represent quasars and galaxies, respectively. The CSS sources are shown as triangles.

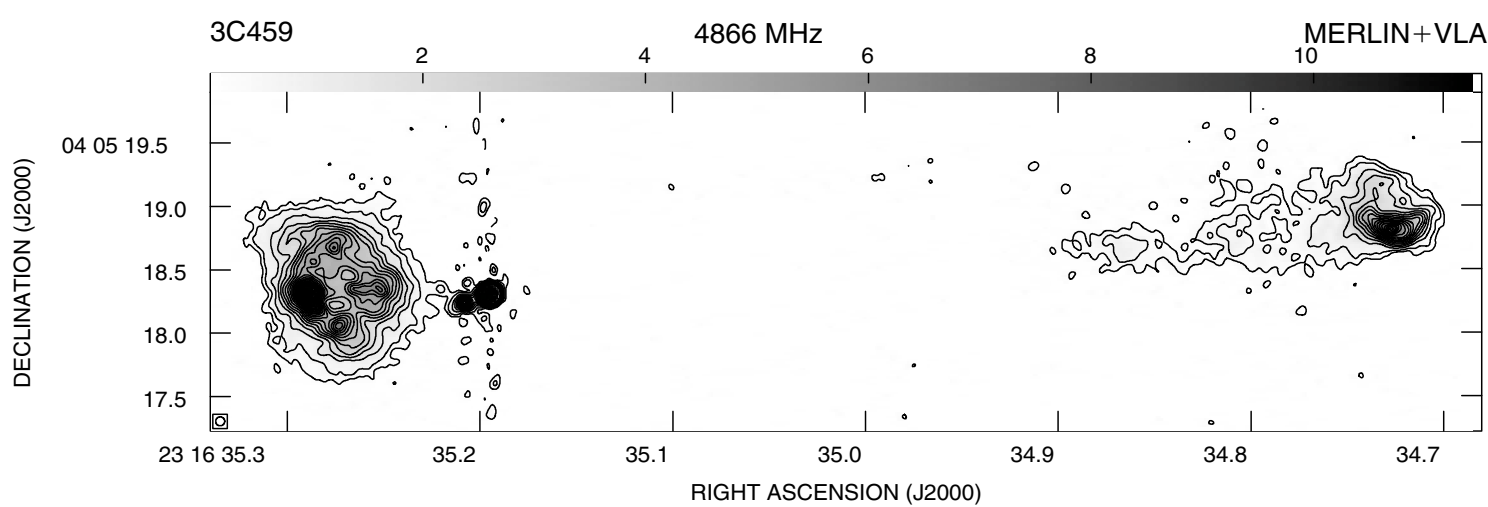

Figure 2 The MERLIN+VLA image of 3C 459 at $4866 \mathrm{MHz}$ with an angular resolution of 70 mas. Peak brightness: $391 \mathrm{mJy} / \mathrm{beam}$; contours: $0.29 \times(-1,1,2,4,6,8,10, \ldots, 36,38,40) \mathrm{mJy} / \mathrm{beam}$.

both radio galaxies and quasars have flatter distributions with higher median values, indicating their evolution in an asymmetric environment (cf. Saikia et al. 2001). The median value of $r_{\mathrm{D}}$ for the CSS galaxies is $\sim 2$ while the value for the larger galaxies is only 1.3. The corresponding values for the quasars are 1.7 and 1.5 respectively. The tendency for smaller sources to be more asymmetric was also reported by Arshakian \& Longair (2000). The deficit of very symmetric quasars is due to their orientation close to the line of sight. In the $r_{\mathrm{D}}-r_{\mathrm{L}}$ diagram for this sample (Figure 1, right), the nearer component is brighter for sources with $r_{\mathrm{L}}<1$, and these have possibly encountered an asymmetric environment. Approximately $65 \%$ of the galaxies have $r_{\mathrm{L}}<1$ compared with $\sim 50 \%$ for quasars, the difference being due to the smaller orientation angles of quasars. Considering only those with $r_{\mathrm{L}}<1$, the median value of $r_{\mathrm{L}}$ is $\sim 0.4$ for CSS sources and close to $\sim 0.6$ for the larger objects, suggesting again that the CSS sources are evolving in a more asymmetric environment.

\subsection{Highly Asymmetric Individual Sources}

In this section, steep spectrum sources with large armlength or separation ratios $\left(r_{\mathrm{D}}>4\right)$ are discussed. One of the most asymmetric CSS sources is the radio galaxy $3 \mathrm{C} 459$, with the ratio of separations of the outer hot spots, $r_{\mathrm{D}}$, being $\sim 5$ and the corresponding flux density ratio, $r_{\mathrm{L}}$, of the oppositely-directed lobes being $\sim 0.45$ and 0.3 at 5 and $1.7 \mathrm{GHz}$ respectively. High-resolution radio images of this source have been produced recently by Thomasson, Saikia, \& Muxlow (2003). Their MERLIN+VLA image of the source is shown in Figure 2. The eastern component, which is brighter, closer to the nucleus and more strongly depolarised, is interacting with denser gas. 3C 459 has a young stellar population and high infrared luminosity, and may have undergone a recent starburst, possibly triggered by the merging of a companion galaxy (Tadhunter et al. 2002 , and references therein). An HST image of the galaxy shows evidence of filamentary structures, which are possibly of tidal origin, and a prominent peak of emission close to that of the nucleus of this N-galaxy, which could be due to a galaxy in the late stages of merging (Thomasson et al. 2003).

From the compilation of symmetry parameters of highluminosity 3CRR and S4 sources (Figure 1), there are only two objects with a separation ratio $>4$, namely $3 \mathrm{C} 254$ and the CSS source B0428+205. 3C 254 is associated with a 
quasar at a redshift of 0.734 with $r_{\mathrm{D}}=6.95$ and $r_{\mathrm{L}}=0.77$ (Owen \& Puschell 1984; P. Thomasson et al., in preparation). Optical line and continuum imaging of this source shows an extended emission line region with the lobe on the nearer side interacting with a cloud of gas (Bremer 1997; Crawford \& Vanderriest 1997). Although the flux density of the nearer lobe is brighter, the ratio is modest, which could be a consequence of relativistic beaming of the hot spot further from the nucleus. The CSS object B0428+205, which has a largest angular size of only 250 mas, is associated with a galaxy at a redshift of 0.219 with $r_{\mathrm{D}}=4.69$ and $r_{\mathrm{L}}=0.16$ (Dallacasa et al. 1995), consistent with a high dissipation of energy on the side where the jet interacts with a dense cloud. Another example of a source with such a high degree of positional asymmetry is the quasar $3 \mathrm{C} 2$ at a redshift of 1.037 for which $r_{\mathrm{D}}=4.7$ and $r_{\mathrm{L}} \sim 0.25$ (Saikia, Salter, \& Muxlow 1987). There is an indication of a radio jet extending from the core to the northern lobe. Depolarisation gradients in both the lobes suggest interaction with the external medium, but the northern lobe seems to be more significantly affected.

In the compilation of McCarthy, van Breugel, \& Kapahi (1991), there are only four galaxies with $r_{\mathrm{D}}>4$, namely, 3C 99, 3C 208.1, 3C 459, and 3C 460. All these sources exhibit a large asymmetry in their flux density ratio, with the brighter lobe being closer to the nucleus. In the case of $3 \mathrm{C} 460$, where they detect significant extended emission line gas, the surface brightness of this gas is much higher on the side of the lobe closer to the nucleus. The radio galaxy $3 \mathrm{C} 99$ is similar to $3 \mathrm{C} 459$ in that it also has a steep spectrum radio core which was resolved by the EVN and shows multiple components. Its value of $r_{\mathrm{D}}=4.8$, while $r_{\mathrm{L}} \sim 0.04$ and 0.03 at 5 and $1.7 \mathrm{GHz}$ respectively, is consistent with the possibility that the jet on the side of the nearer lobe is interacting with denser gas. Optical spectroscopic observations along the axis of the source do indeed show that the gas on the side of the nearer component is blueshifted while that on the opposite side is redshifted relative to the galaxy. The blueshifted gas is possibly being pushed towards us by interaction with the radio jet (Mantovani et al. 1990).

\subsection{Flux Density Asymmetry for a Larger Sample}

The flux density ratio alone could be examined for a larger sample of sources since here one does not require the detection of a radio core. Towards this end, a sample of sources of intermediate strength selected from the $408 \mathrm{MHz}$ Bologna B2.1 catalogue (Colla et al. 1970) has been observed with the VLA A-array (Saikia et al. 2002). The sample consists of 52 objects which satisfy the following criteria. They lie within the region, $23^{\mathrm{h}}$ $30^{\mathrm{m}}<\mathrm{RA}(\mathrm{B} 1950)<2^{\mathrm{h}} 30^{\mathrm{m}}$ and $29^{\circ} 18^{\prime}<\operatorname{dec}(\mathrm{B} 1950)$ $<34^{\circ} 02^{\prime}$, with their flux densities in the range $0.9 \leq$ $\mathrm{S}_{408}<2.5 \mathrm{Jy}$. Of these 52, 19 candidate CSS sources have been observed with the VLA A-array at $4835 \mathrm{MHz}$. A few of the radio images are shown in Figure 3. In addition to this sample, the CSS sources from the B3+VLA sample observed by Fanti et al. (2001) and the 3CRR sources have also been considered.

The B2 sample: Considering the steep spectrum, double-lobed B2 sources, the 'small-source' sample is defined to consist of those with an LAS $<10$ arcsec. The remaining objects constitute the 'large-source' sample. The median angular size of the 'small-source' sample is $\sim 4$ arcsec compared with $\sim 40$ arcsec for the larger sources. The flux density ratio of the oppositely directed lobes, $r_{\mathrm{L}}$, has been estimated from either our observations or from those in the literature. $r_{\mathrm{L}}$ ranges from $\sim 1.4$ to 20 for the small-source sample, and has a median value of $\sim 2$, while for the large-source sample, $r_{\mathrm{L}}$ ranges from $\sim 1.0$ to 7 , with a median value of $\sim 1.4$ (Figure 4 , left).

A significant fraction of the small sources show large asymmetries. Defining a very asymmetric source to be one with $r_{\mathrm{L}}>5$, we find that 5 of the 12 small-sized objects $(\sim 40 \%)$ are highly asymmetric, while only one of the 22 $(\sim 5 \%)$ objects in the large-source B2 sample has $r_{\mathrm{L}}>5$.

The 3CRR sample: It is of interest to compare this with earlier studies of the flux density ratio for sources in the well-known 3CRR sample. For the 3CRR sources studied by Saikia et al. (2001), which were confined to the high-luminosity FR II sources, the median values of $r_{\mathrm{L}}$ for the CSS sources and the large sources are $\sim 2$ and 1.8 respectively (Figure 4, right). This is similar to the values for the B2 sources. The distribution for the CSS sources again appears to have a number of very asymmetric sources with three of the 13 sources $(\sim 23 \%)$ having a value of $r_{\mathrm{L}}>5$, whereas only five of the $75(\sim 7 \%)$ non-CSS 3CRR sources exhibit such a high degree of asymmetry.

The B3 CSS sources: The above result has also been compared with that derived from a sample of CSS sources selected from the B3-VLA sample with $S_{408} \geq 0.8 \mathrm{Jy}$ (Fanti et al. 2001). Considering all the doubles, plus the collinear triple, 0744+464, which has a weak central component, the median value of $r_{\mathrm{L}}$ for the B3-VLA CSS sample is $\sim 2.4$, with eight of the 39 objects $(\sim 21 \%)$ having a value of $r_{\mathrm{L}}>5$ (Figure 5, left). This is consistent with the results for the $\mathrm{B} 2$ and $3 \mathrm{CRR}$ samples.

Considering the B2, 3CRR, and B3-VLA samples together, a Kolmogorov-Smirnov test shows the distributions for the CSS and larger sources to be different at a confidence level of greater than $99 \%$. However, if the most asymmetric objects, i.e., those with $r_{\mathrm{L}}>5$, are excluded, the distributions for the rest of the sources are not significantly different. The fraction of such asymmetric sources for the combined sample is $25 \%$ for the CSS sources and $6 \%$ for the larger sources. The increased fraction for the CSS sources is possibly due to interaction of one of the jets with a dense cloud on one side of the nucleus. These density asymmetries might be intimately related to the infall of gas which fuels the radio source. Assuming the typical distances of these clouds of gas from the nucleus of the galaxy to be about $5-10 \mathrm{kpc}$, the covering factor estimated from the fraction of very asymmetric sources suggests that the sizes of the 'clouds' are similar to those 

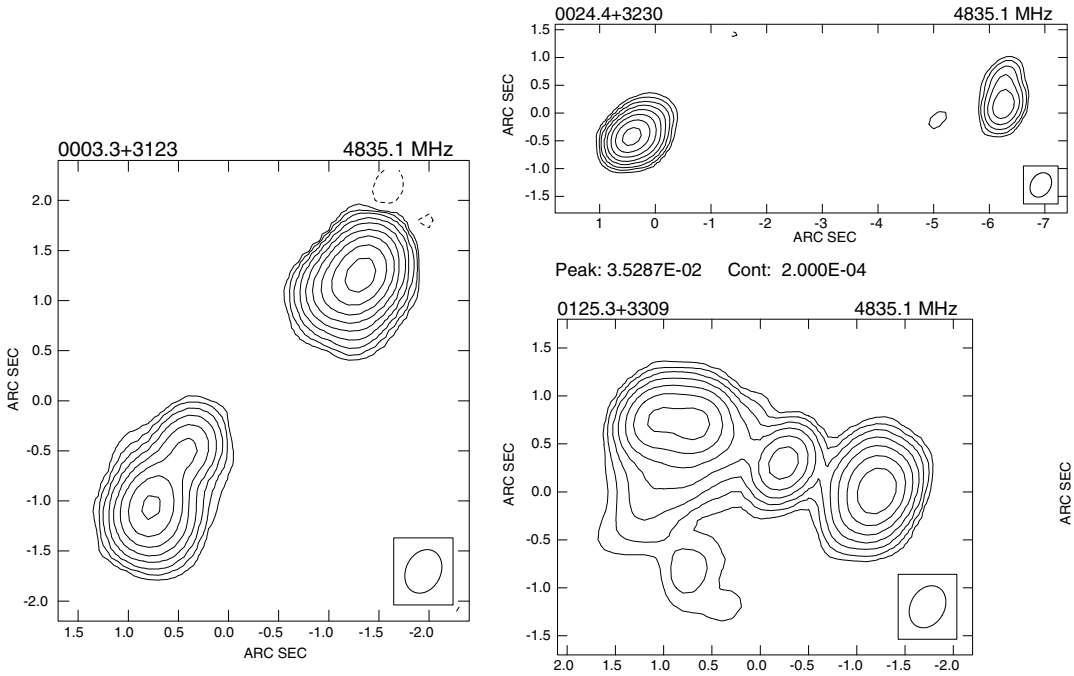

Peak: 3.5287E-02 Cont: $2.000 \mathrm{E}-04$
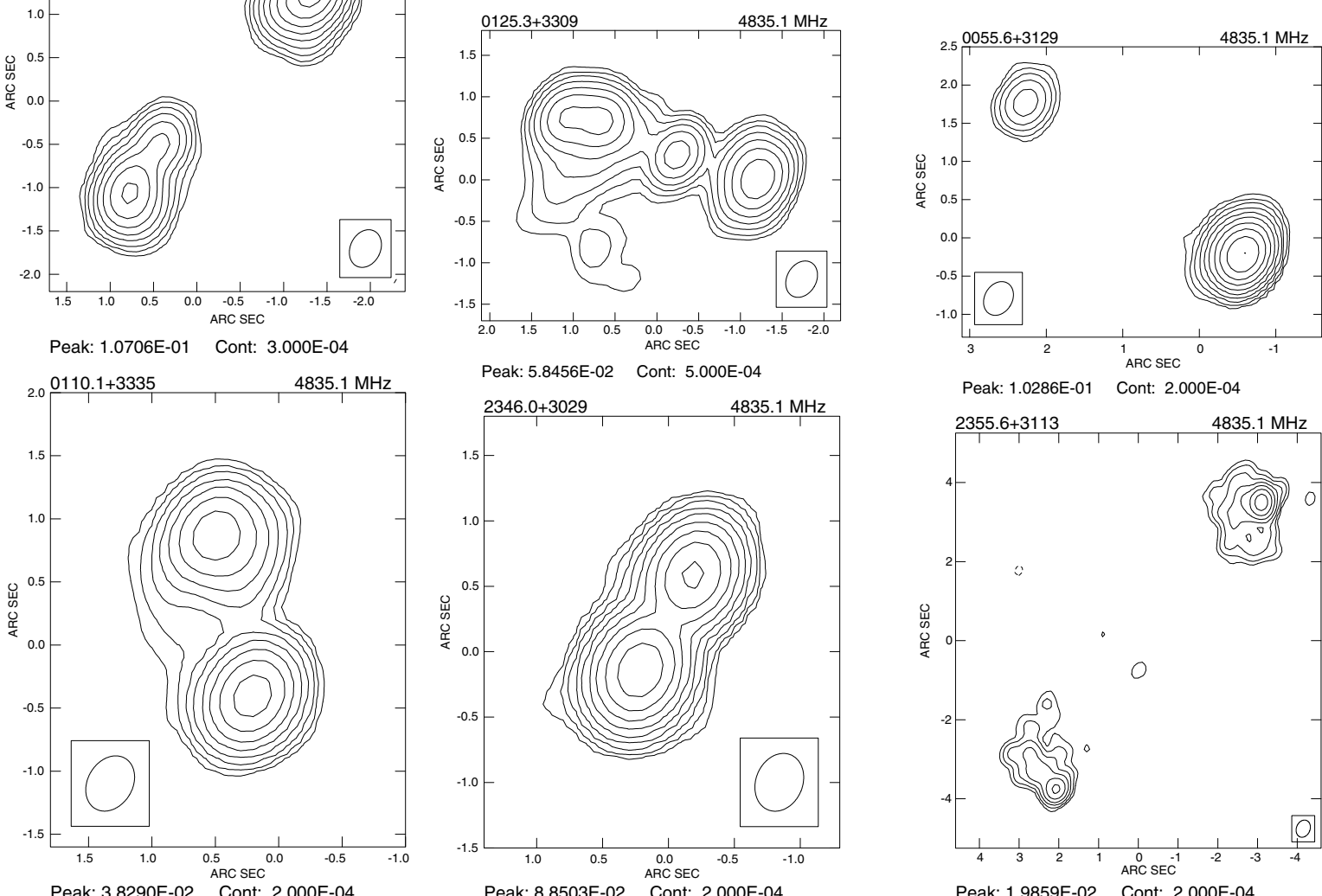

Peak: 1.0286E-01 Cont: 2.000E-04

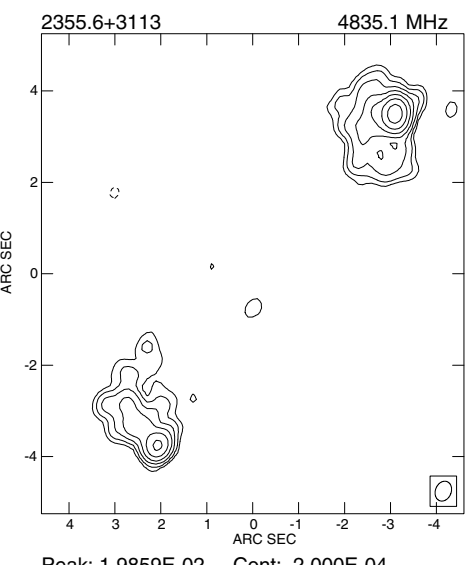

Figure 3 VLA A-array images of some of the candidate CSS objects from the B2 sample of sources. The contour levels for all the images are $-1,1,2,4,8,16, \ldots$ times the first contour level. The peak brightness in the image and the level of the first contour in units of Jy/beam are given below the images. The half-power ellipse of the restoring beam is shown in one of the lower corners of each image.

of dwarf galaxies (e.g. Swaters 1999). There could, of course, be a larger number of smaller clouds. However, if the clouds are too small or are of relatively small mass, they are unlikely to significantly affect the propagation of the jet (e.g. Wang, Wiita, \& Hooda 2000).

The flux density ratio-redshift diagram: It is also of interest to enquire whether the brightness asymmetries of CSS sources depend on cosmic epoch because of a larger incidence of interactions and mergers in the past (Ellis et al. 2000). The $r_{\mathrm{L}}$-redshift diagram for the 43 CSS sources with measured or estimated redshifts from the combined sample (Figure 5, right) shows no significant dependence on redshift. This suggests that although interactions and mergers may increase with redshift globally, the flux density asymmetries in the CSS objects, which are possibly young and still being fuelled by the infall of gas, are similar at different redshifts.

\subsection{Polarisation Measurements}

Evidence of the gas which fuels the radio source may also be probed via polarisation measurements (cf. Saikia \&
Salter 1988; van Breugel et al. 1992; Akujor \& Garrington 1995). In a double-lobed radio source, the component interacting with the dense gas should normally both exhibit a higher rotation measure (RM) and also depolarise more rapidly than the component advancing through the more tenuous medium. Although there have been very few studies of RM determinations in CSS and GPS objects, partly due to their low levels of polarisation, such studies have sometimes revealed striking differences in the RM on opposite sides of the nucleus. Detailed polarisation observations of 3C 147 show huge differential RMs between the two oppositely directed lobes, suggesting their evolution in an asymmetric environment (Junor et al. 1999). A similar situation is also seen in the quasar $0725+147$ (Mantovani et al. 1994). These asymmetries in the central regions of active galaxies must be intimately related to the supply of fuel to the central engine in these objects, possibly due to interactions with companion galaxies.

In the absence of RM determinations, one can also examine the asymmetry in the polarisation of the two oppositely directed lobes of radio emission. A sample 

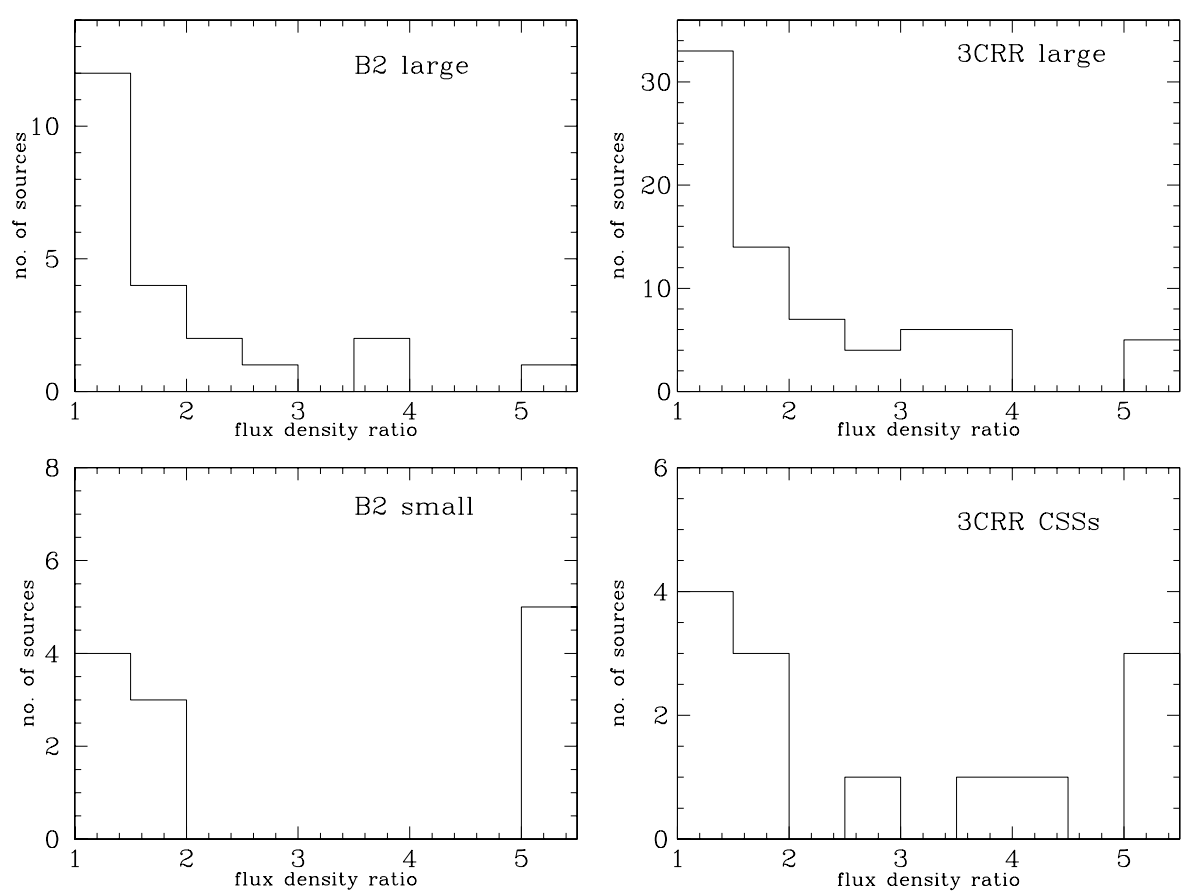

Figure 4 The distributions of the flux density ratio, $r_{\mathrm{L}}$ for the B2 and 3CRR sources discussed in the text. All the sources with $r_{\mathrm{L}}>5$ have been placed in the last bin.
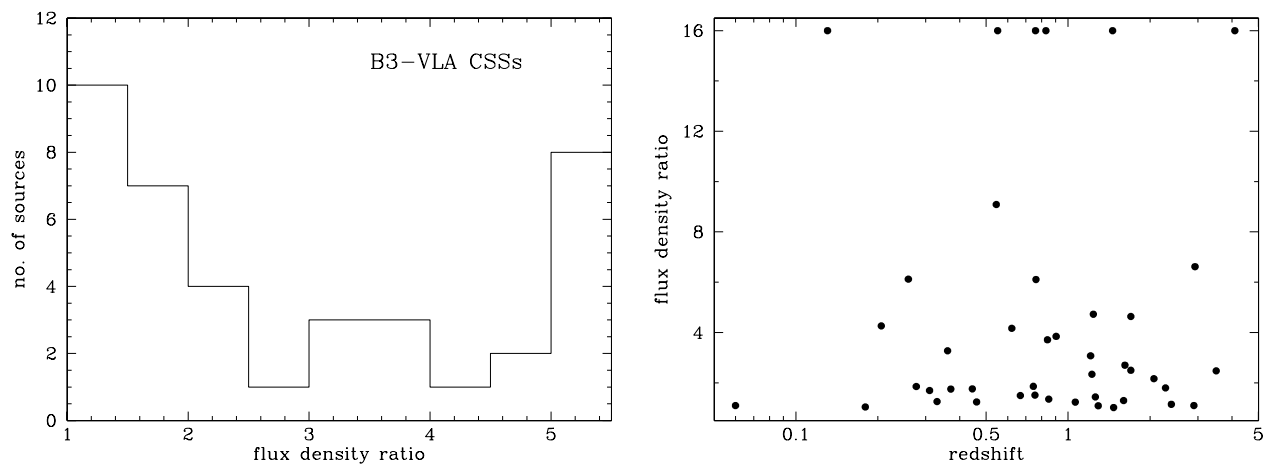

Figure 5 Left: The distribution of $r_{\mathrm{L}}$ for the B3-VLA CSS sources. All the sources with $r_{\mathrm{L}}>5$ have been placed in the last bin. Right: The $r_{\mathrm{L}}$-redshift diagram for the B2, 3CRR, and B3 CSS sources discussed in the text.

of CSS sources has been observed with the VLA to determine the asymmetry in the polarisation of the oppositely-directed lobes (D.J. Saikia et al., in preparation). Two of these sources are presented in Figure 6, and their polarisation parameters are listed in Table 1. The median value of the ratio of the degree of polarisation of the oppositely-directed lobes at $\lambda 6$ or $3.6 \mathrm{~cm}$ is $\sim 6$ for the CSS objects compared with $\sim 1.5$ for the larger sources. A Kolmogorov-Smirnov test shows the two populations to be different at a significance level of $>99.9 \%$. This high degree of polarisation asymmetry is also likely to be due to interactions with dense clouds of gas which could either compress magnetic fields, increasing the degree of polarisation, or else depolarise the emission. The degree of polarisation asymmetry is much higher than due to the Laing-Garrington effect (Laing 1988; Garrington et al. 1988). In a number of cases, it is the jet side which is more strongly depolarised (e.g. 3C 459) or has a much higher
RM (e.g. 3C 147). Also, the typical core radii of the halos which cause the Laing-Garrington effect is $\sim 100 \mathrm{kpc}$, which will cause only marginal polarisation asymmetry on the scale of the CSS objects (cf. Garrington \& Conway 1991).

\section{Numerical Simulation}

We have attempted to understand the evolution of CSS sources through an asymmetric environment in the nuclear region by calculating analytical models (Saikia et al. 1996) as well as performing two- and three-dimensional simulations (Figure 7) of the propagation of jets through asymmetric environments (Jeyakumar et al. in preparation). In this study, intrinsically similar jets with external Mach numbers of 26.0 propagate through ambient media with different gas distributions in the inner $5 \mathrm{kpc}$; at first the bow shocks run substantially ahead of the jet working surfaces. The jet propagating through the less dense 

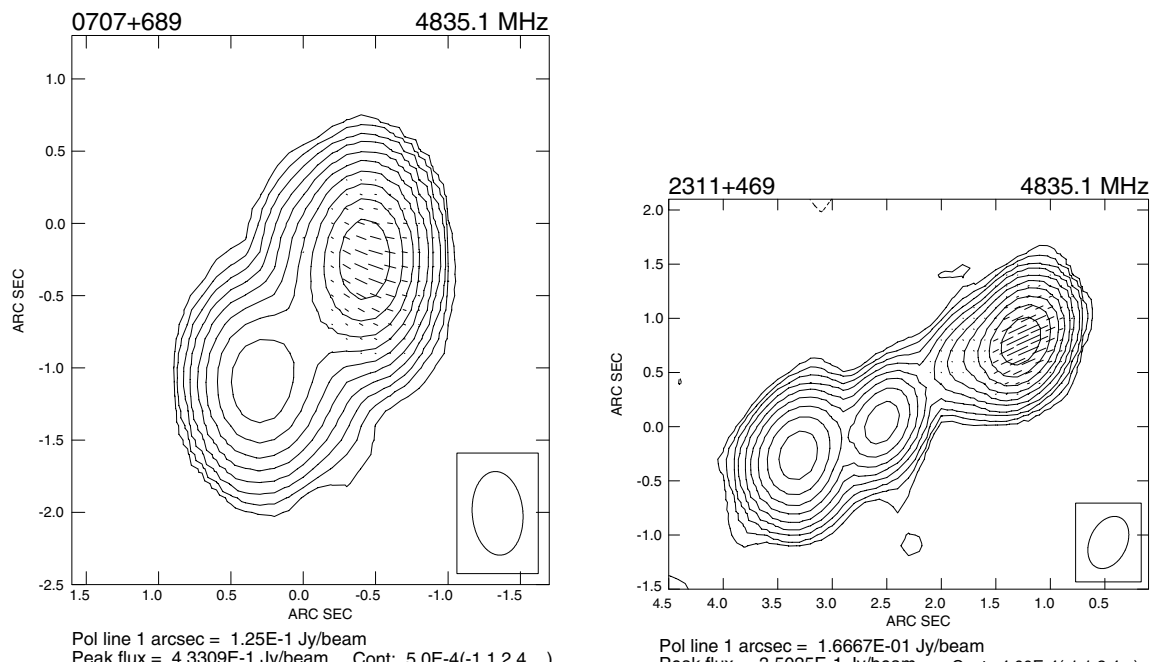

Pol line 1 arcsec $=1.6667 \mathrm{E}-01 \mathrm{Jy} / \mathrm{beam}$

Peak flux = 4.3309E-1 Jy/beam Cont: $5.0 \mathrm{E}-4(-1,1,2,4 \ldots)$

Peak flux $=3.5025 \mathrm{E}-1 \mathrm{Jy} / \mathrm{beam}$ Cont: $4.00 \mathrm{E}-4(-1,1,2,4 \ldots)$

Figure 6 VLA A-array images of two CSS objects from the S4 sample, 0707+689 and 2311+469, illustrating the high degree of polarisation asymmetry.

Table 1. The polarisation parameters

\begin{tabular}{lcclccc}
\hline Source & Opt. Id. & $z$ & \multicolumn{1}{c}{ Resolution } & $m_{\text {west }}$ & $m_{\text {east }}$ & $m_{\text {west }} / m_{\text {east }}$ \\
\hline B0707+689 & Q & 1.141 & 0. . $^{\prime \prime} 58 \times 0 . .^{\prime \prime} 35$ along $5^{\circ}$ & 2.61 & $<0.23$ & $>11.3$ \\
B2311+469 & Q & 0.742 & $0 .^{\prime \prime} 51 \times 0 .^{\prime \prime} 34$ along $155^{\circ}$ & 9.72 & $<0.20$ & $>48.6$ \\
\hline
\end{tabular}
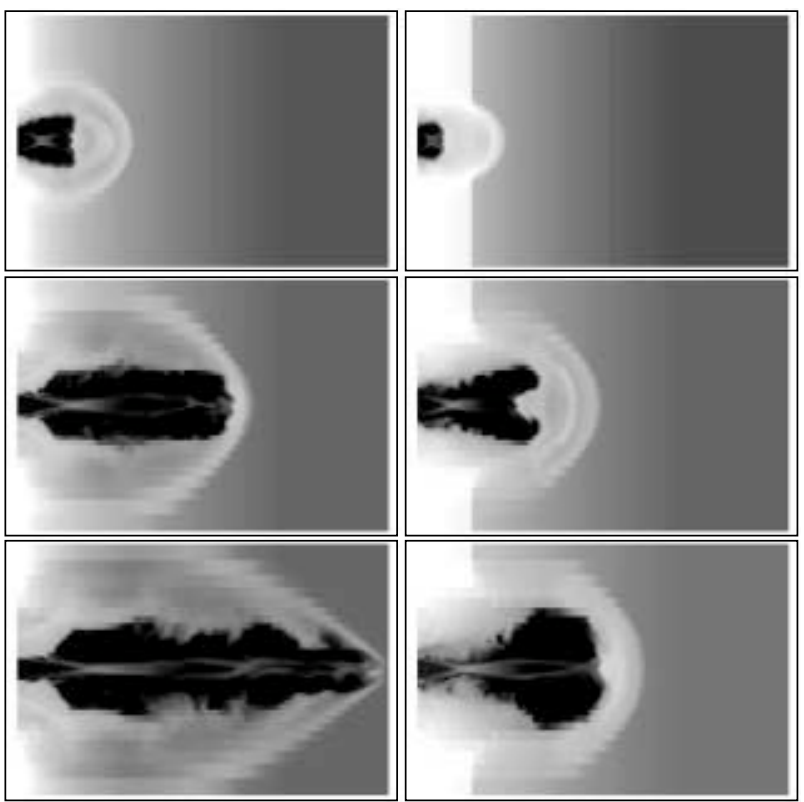

Figure 7 The logarithm of the density (with white highest and black lowest) is shown for 3-D simulations with identical jet parameters passing through a smooth decline in external gas density (left) and a more gradual decline, followed by an abrupt drop to an identical gas density after $5 \mathrm{kpc}$ (right). The times correspond to $1.3 \times 10^{5} \mathrm{yr}$ (top), $4.8 \times 10^{5} \mathrm{yr}$ (middle), and $8.6 \times 10^{5} \mathrm{yr}$ (bottom).

material naturally emerges faster, and the value of $r_{\mathrm{D}}$ rises quickly; it later declines as the jets traverse identical media further out (Figure 8). The numerical simulations qualitatively agree with the analytical models and support

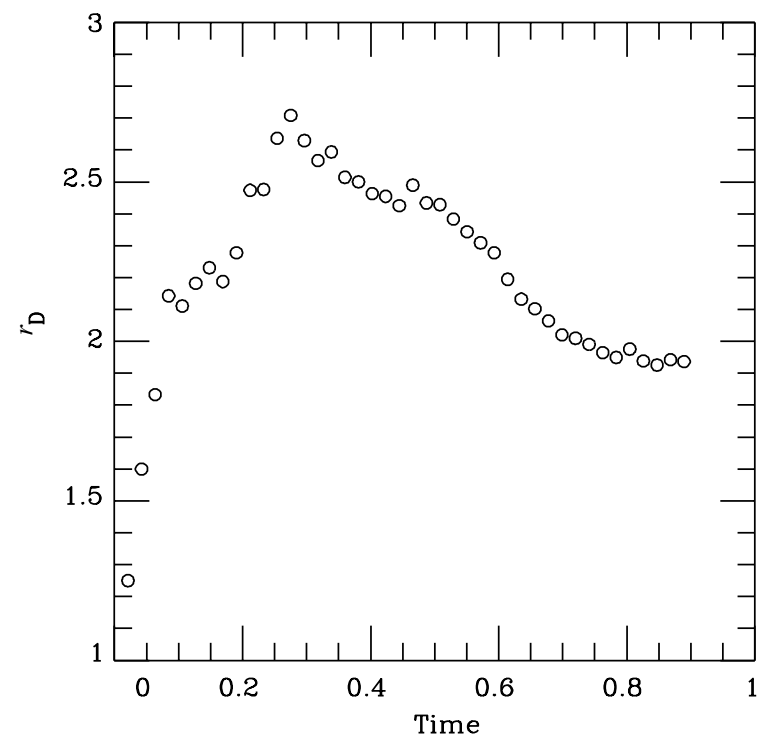

Figure 8 The variation of $r_{\mathrm{D}}$ estimated using the bow shock as a function of time scaled to units of Myr.

the hypothesis that CSS sources show greater asymmetries than do FR IIs predominantly because ambient gas asymmetries (the AGN fuel?) are concentrated in the inner portions of the galaxies.

\section{References}

Akujor, C. E., \& Garrington, S. T. 1995, A\&AS, 112, 235

Arshakian, T. G., \& Longair, M. S. 2000, MNRAS, 311, 846

Bremer, M. N. 1997, MNRAS, 284, 126 
Carvalho, J. C. 1985, MNRAS, 215, 463

Colla, G., et al. 1970, A\&AS, 1, 281

Crawford, C. S., \& Vanderriest, C. 1997, MNRAS, 285, 580

Dallacasa, D., Fanti, C., Fanti, R., Schilizzi, R. T., \& Spencer, R. E. 1995, A\&A, 295, 27

De Young, D. S. 1997, ApJ, 490, 55L

Ellis, R. S., Abraham, R. G., Brinchmann, J., \& Menanteau, F. 2000, A\&G, No. 2, 10

Fanti, C., Fanti, R., Dallacasa, D., Schilizzi, R. T., Spencer, R. E., \& Stanghellini, C. 1995, A\&A, 302, 317

Fanti, C., Pozzi, F., Dallacasa, D., Fanti, R., Gregorini, L., Stanghellini, C., \& Vigotti, M. 2001, A\&A, 369, 380

Garrington, S. T., \& Conway, R. G. 1991, MNRAS, 250, 198

Garrington, S. T., Leahy, J. P., Conway, R. G., \& Laing, R. A. 1988, Nature, 331, 147

Hernquist, L., \& Mihos, J. C. 1995, ApJ, 448, 41

Jeyakumar, S., \& Saikia, D. J. 2000, MNRAS, 311, 397

Junor, W., Salter, C. J., Saikia, D. J., Mantovani, F., \& Peck, A. B. 1999, MNRAS, 308, 955

Laing, R. A. 1988, Nature, 331, 149

Mantovani, F., Junor, W., Fanti, R., Padrielli, L., \& Saikia, D. J. 1994, A\&A, 292, 59

Mantovani, F., Saikia, D. J., Browne, I. W. A., Fanti, R., Muxlow, T. W. B., \& Padrielli, L. 1990, MNRAS, 245, 427

McCarthy, P. J., van Breugel, W., \& Kapahi, V. K. 1991, ApJ, 371,478

Murgia, M., Fanti, C., Fanti, R., Gregorini, L., Klein, U., Mack, K.-H., \& Vigotti, M. 1999, A\&A, 345, 769
O'Dea, C. P. 1998, PASP, 110, 493

Owen, F. N., \& Puschell, J. J. 1984, AJ, 89, 932

Owsianik, I., \& Conway, J. E. 1998, A\&A, 337, 69

Perucho, M., \& Martí, J. M. 2002, ApJ, 568, 639

Readhead, A., Taylor, G., Pearson, T., \& Wilkinson, P. 1996, ApJ, 460,634

Saikia, D. J., \& Salter, C. J., 1988, ARA\&A, 26, 93

Saikia, D. J., Salter, C. J., \& Muxlow, T. W. B. 1987, MNRAS, 224, 911

Saikia, D. J., Jeyakumar, S., Wiita, P. J., \& Hooda, J. S. 1996, in The Second Workshop on GPS and CSS Sources, ed. I. A. G. Snellen et al. (Leiden: Leiden Observatory), 252

Saikia, D. J., Jeyakumar, S., Salter, C. J., Thomasson, P., Spencer, R. E., \& Mantovani, F. 2001, MNRAS, 321, 37

Saikia, D. J., Thomasson, P., Spencer, R. E., Mantovani, F., Salter, C. J., \& Jeyakumar, S. 2002, A\&A, 391, 149

Snellen, I. A. G., Schilizzi, R. T., Miley, G. K., de Bruyn, A. G., Bremer, M. N., \& Röttgering, H. J. A. 2000, MNRAS, 319, 445

Swaters, R. A. 1999, PhD thesis, Rijksuniversiteit Groningen

Tadhunter, C., Dickson, R., Morganti, R., Robinson, T. G., Wills, K., Villar-Martin, M., \& Hughes, M. 2002, MNRAS, 330, 997

Taylor, G. B., Marr, J. M., Pearson, T. J., \& Readhead, A. C. S. 2000, ApJ, 541, 112

Thomasson, P., Saikia, D. J., \& Muxlow, T. W. B. 2003, MNRAS, in press

van Breugel, W. J. M., Fanti, C., Fanti, R., Stanghellini, C., Schilizzi, R. T., \& Spencer, R. E. 1992, A\&A, 256, 56

Wang, Z., Wiita, P. J., \& Hooda, J. S. 2000, ApJ, 534, 201 